\title{
ALGUMAS ESTRUTURAS DO CARBONO ELEMENTAR E SUA IMPORTÂNCIA PARA O DESENVOLVIMENTO E SOBERANIA DO BRASIL
}

\author{
Aldo J. G. Zarbin*,a,® e Elisa S. Orth ${ }^{a,(1)}$ \\ aDepartamento de Química, Universidade Federal do Paraná, 81531-980 Curitiba - PR, Brasil
}

Recebido em 02/08/2019; aceito em 03/10/2019; publicado na web em 12/11/2019

\begin{abstract}
SOME ELEMENTAL CARBON STRUCTURES AND THEIR IMPORTANCE ON THE DEVELOPMENT AND SOVEREIGNITY OF BRAZIL. Undoubtedly, the chemical element carbon is strategic for the development of any country and herein we show some specific impacts in the economical, industrial and scientific sovereignty of Brazil, with emphasis on $\mathrm{sp}^{2}$ carbn structures, namely graphite, carbon black, carbon nanotubes and graphene. Brazil comprises the second greatest natural reserve of graphite worldwide and ranks as third in world production. Carbon black also has important industrial impact in Brazil, especially in the automobile industry, specifically in tires. Some of world leading companies in the production of carbon black are installed in Brazil. Finally, carbon nanotubes and graphene are addressed as the next generation of materials that have and will have great impact in the next years. Their impressive properties have enabled innumerous commercial products worldwide and Brazil already stands out with some innovative initiatives. Indeed, Brazil has installed capacity in universities and research institutes to meet and foster demands in the area, and can be competitive worldwide.
\end{abstract}

Keywords: carbon; graphite; graphene; carbon nanotubes; carbon black.

\section{INTRODUÇÃO}

Definir "materiais estratégicos" não é uma tarefa fácil, uma vez que diferentes perspectivas podem ser associadas a esse termo. De forma bastante resumida, um material estratégico é aquele pelo qual algum planejamento estratégico é diretamente dependente, e sua ausência pode acarretar um dano direto a esse planejamento. No que se refere a um material estratégico para uma nação, esse planejamento diz respeito a aspectos econômicos, políticos, sociais ou militares (segurança): materiais que geram riqueza (de forma direta ou indireta), que garantem soberania, que possibilitam o bem estar da população quando incorporados em produtos (ou quando estão associados a serviços), e que de alguma forma auxiliam na defesa da nação. É interessante notar que diferentes nações possuem estágios diferentes de desenvolvimento econômico, político e social, o que faz com que o que é considerado estratégico para uma nação, pode não ser para outra.

Trazendo a definição para o contexto dos elementos químicos, pode-se propor que um elemento estratégico é aquele pelo qual sua presença é fundamental para a existência de um material estratégico. Esse artigo tem como foco o elemento químico carbono como estratégico para o Brasil, com ênfase no carbono elementar, e mais especificamente em estruturas de carbono elementar derivativas do grafite, com hibridização $\mathrm{sp}^{2}$. Com esse afunilamento, os autores optam por quatro estruturas onde o elemento é o próprio material, sendo duas delas bastante consolidadas e com um peso significativo na economia do país (grafite e negro de fumo), e duas promessas que certamente terão um grande impacto nos próximos anos (nanotubos de carbono e grafeno). Com essa diretriz, o diamante (que é um alótropo do carbono com hibridização $\mathrm{sp}^{3}$ ), o carvão, os fulerenos e as diferentes formas de carbono amorfo não serão considerados, embora sua importância na economia e política do Brasil não possa ser negligenciada. Da mesma forma, não serão discutidos compostos, ou seja, materiais ou moléculas onde o carbono faz parte da estrutura/ composição (como os diferentes carbonatos e compostos orgânicos

*e-mail: aldozarbin@ufpr.br aplicados em fármacos, por exemplo), e tampouco serão abordados aspectos relacionados à chamada economia de baixo carbono, e as vantagens econômicas potencialmente advindas de uma baixa emissão de $\mathrm{CO}_{2}$.

Grafite, negro de fumo, nanotubos de carbono e grafeno são estruturas baseadas em diferentes arranjos de átomos de carbono com hibridização $\mathrm{sp}^{2}$. Um detalhamento aprofundado sobre as características estruturais de cada um desses materiais pode ser encontrado na literatura. ${ }^{1-4} \mathrm{O}$ grafite é formado por átomos de carbono com hibridização $\mathrm{sp}^{2}$, ligados a outros três átomos idênticos no plano, formando estruturas planares representadas por hexágonos de átomos de carbono similares a uma colmeia. O empilhamento dessas estruturas bidimensionais por atração de van der Waals, com uma separação de $0,335 \mathrm{~nm}$ entre elas, origina a estrutura do grafite. O grafeno consiste exatamente em uma folha individual do grafite, sendo, portanto, um material bidimensional, crescendo nas dimensões a e b do plano, e com um único átomo de espessura na direção c. A estrutura dos nanotubos de carbono pode ser idealizada como se fosse uma folha de grafeno enrolada, formando tubos de diâmetros nanométricos, que têm propriedades dependentes tanto do diâmetro como da forma pela qual esses tubos se enrolam. Além disso, diferentes folhas podem ser enroladas de forma concêntrica, separadas por distâncias similares à separação das folhas de grafeno no grafite, originando os nanotubos de carbono de paredes múltiplas. E o negro de fumo (do inglês carbon black) é caracterizado por partículas grafíticas esféricas, com diâmetros individuais variando na faixa de 10 a $100 \mathrm{~nm}$, que normalmente se agregam formando estruturas elipsoides maiores, com frações amorfas e cristalinas, sendo os domínios cristalinos normalmente presentes na superfície das partículas. Grupos oxigenados como carboxilas, hidroxilas, fenóis, quinonas, aldeídos, dentre outros, geralmente estão presentes na superfície dessas partículas, em quantidades variadas, influenciando fortemente suas propriedades.

\section{GRAFITE}

O grafite (ou a grafita) é um mineral natural, que pode também ser preparado sinteticamente através de tratamentos térmicos em 
altas temperaturas de carbono amorfo ou outros precursores, como determinados tipos de polímeros. Dentre suas propriedades que os tornam úteis para diferentes tipos de aplicação, pode-se citar uma elevada condutividade elétrica e térmica; sua inércia química, sendo um material bastante resistente à corrosão e ataques químicos; sua inércia ao meio ambiente; sua maciez e propriedades de lubrificante no estado sólido, que são melhoradas em altas temperaturas e pressões; sua capacidade de acomodar íons, átomos e moléculas entre suas camadas, em reações de intercalação; seu alto ponto de fusão $\left(3650{ }^{\circ} \mathrm{C}\right)$. Dentre as aplicações industriais do grafite, destacam-se seu uso em cerâmicas e materiais refratários; em peças e componentes como pastilhas de freios e escovas para motores elétricos; como aditivos em metalurgia; como eletrodos de baterias; como lubrificantes; como aditivos em polímeros (retardante de chama, aumento de condutividade), dentre outras.

$\mathrm{Na}$ sua ocorrência natural, existem três formas de grafite comercial, que diferem entre si pelo tamanho das partículas, pureza e tipo de contaminantes: ${ }^{5}$ o chamado grafite flake, que possui cerca de $85 \%$ de carbono; o grafite amorfo, que possui entre $65 \%$ e $85 \%$ de carbono, e apesar do nome não é um material amorfo; e o grafite veio (ou lump), que possui aproximadamente $95 \%$ de carbono. O Brasil é o terceiro maior produtor mundial de grafite, ${ }^{5}$ ficando atrás da China e Índia. Segundo dados do Departamento Nacional de Produção Mineral (DNPM), ${ }^{5}$ em 2015 a produção mundial de grafite foi de 1,19 milhões de toneladas, sendo $65,5 \%$ desse total produzido pela China, $14,3 \%$ pela Índia e $6,9 \%$ pelo Brasil. Além desses três grandes produtores, destacam-se Turquia $(2,7 \%)$, Coreia do Norte $(2,5 \%)$ e Canadá (2,5\%), além de países que produzem em menor escala como Rússia, México, Ucrânia e Madagascar. É interessante notar que $31,4 \%$ de todo o grafite natural existente no Planeta encontra-se no Brasil (o que corresponde à segunda maior reserva de grafite natural), concentrado principalmente nos estados de Minas Gerais, Ceará e Bahia. ${ }^{5}$ A maior produtora brasileira é a empresa Nacional de Grafite Ltda, responsável por $84 \%$ da produção brasileira de 2015 . $^{5}$

Os dados apresentados acima não deixam dúvidas quanto à importância estratégica do grafite para o Brasil. Entretanto, é fundamental que o país olhe para essa riqueza não somente como uma commodity (cujo valor de mercado flutua de acordo com indicadores voláteis e de difícil controle), mas que o parque industrial brasileiro consiga transformá-lo em bens manufaturados de alto valor agregado. Com relação a essa afirmação, são sintomáticos alguns dados da balança comercial brasileira: o relatório do DNPM relativo ao ano de 2015 descreve que o Brasil exportou um total de 20.798 toneladas de bens primários relacionados ao grafite, ${ }^{5}$ rendendo um faturamento de US\$29,4 milhões, sendo que Estados Unidos (32\%), Alemanha (26\%), Bélgica (17\%), Japão (13\%) e Índia (7\%) foram os principais países destino. Além disso, foram exportadas 4.401 toneladas em produtos manufaturados cuja composição fosse predominantemente a base de grafite, gerando US\$14,5 milhões, com Estados Unidos (20\%), Argentina (12\%), Peru (10\%), Chile (10\%) e Tailândia (9\%) sendo os principais países compradores. Uma conta simples indica um faturamento de US\$ 43,9 milhões devido a exportações diretamente relacionadas ao grafite. Nesse mesmo ano, as importações de produtos primários foram de somente 518 toneladas (US\$ 1,4 milhões), enquanto que as importações de manufaturados de grafite foram de 25.347 toneladas, vindos dos Estados Unidos (33\%) México (18\%), Espanha (11\%), Áustria (6\%) e China (6\%), totalizando um dispêndio de US\$116,2 milhões. O total que o Brasil gastou com importações relacionadas ao grafite foi de US\$117,6 milhões, o que corresponde a um déficit na balança comercial de US\$ 72,3 milhões. Esses dados não são aceitáveis para o terceiro maior produtor de grafite do mundo. A relação com os Estados Unidos é bastante representativa desse cenário: de acordo com o relatório do United States Geological
Survey (USGS) em 2017, ${ }^{6}$ não existe nenhuma mina em produção do grafite nos EUA, portanto, toda demanda daquele país é importada (em 2017 foram 37\% da China, 29\% do México, 17\% do Canadá e somente $9 \%$ do Brasil). Entretanto, o Brasil importa dos EUA produtos manufaturados a base de grafite, o que significa um pronunciado aumento no seu valor agregado. Em 2015 esse valor correspondeu a US\$ 38,35 milhões, valor muito superior ao vendido aos EUA pelo Brasil na forma de bem primário: US\$ 9,41 milhões.

O consumo mundial de grafite vem crescendo desde $2013 .{ }^{6}$ Embora a principal utilização do grafite ainda seja na indústria de refratários, existe uma expectativa de demanda crescente nas baterias, tanto de íons lítio (e recentemente de íons sódio e potássio), quanto em células a combustível, devido ao advento de carros elétricos e híbridos, além da demanda crescente por celulares, tablets e notebooks: utilização em alta tecnologia, aumentando seu valor agregado.

\section{NEGRO DE FUMO (“CARBON BLACK”)}

Um dos produtos mais utilizados globalmente, o negro de fumo é produzido em grandes quantidades através da combustão incompleta de hidrocarbonetos. Trata-se, portanto, de um produto da indústria petroquímica, com um consumo global estimado em 13,2 milhões de toneladas métricas em 2015, em um mercado estimado em US\$ 13,7 bilhões. ${ }^{2,7} \mathrm{O}$ negro de fumo é usado como pigmento preto em tintas, toners, como agente antiestática e, principalmente, como carga em polímeros. ${ }^{8}$ Estima-se que $70 \%$ de todo negro de fumo produzido globalmente seja utilizado como aditivo em borracha para pneus, visando pigmentação, reforço mecânico e condução de calor, o que aumenta significativamente o tempo de vida dos pneus. ${ }^{2}$ Valores entre 30 e $35 \%$ da massa total de um pneu são devidos ao negro de fumo. As projeções de mercado para o negro de fumo é que seu consumo continuará crescente até 2025, atingindo a marca de 15 milhões de toneladas métricas. ${ }^{7}$

Os maiores consumidores, e também produtores de negro de fumo, são a China e a Índia. No Brasil, a produção de negro de fumo se iniciou em 1958, com a Copebras - Companhia Petroquímica Brasileira - produzindo 50 mil toneladas anuais na sua fábrica em Cubatão-SP. ${ }^{9}$ Hoje a fábrica pertence à Aditya Birla Group, líder no mercado de negro de fumo na América Latina, ${ }^{9}$ que juntamente com a unidade de Camaçari - BA, tem a capacidade de produção de 267 mil toneladas/ano. ${ }^{9}$ Além da Birla, outros produtores de Negro de Fumo no Brasil são as empresas Cabot (Mauá-SP) e Orion Carbons (Paulínia-SP).

O negro de fumo é um produto cujo mercado está intimamente ligado à indústria automobilística, sendo, portanto, fortemente afetado pelas flutuações na economia. Outros setores, entretanto, vêm com demanda crescente para a utilização de negro de fumo, principalmente como aditivos em diferentes artefatos de borracha (baldes, sacos de lixo, plásticos de engenharia, fios, cabos, etc). Diferentes aplicações requerem tipos/qualidades/estruturas diferentes de negros de fumo, que estão disponíveis atualmente no mercado.

\section{NANOTUBOS DE CARBONO E GRAFENO}

O advento dos nanotubos de carbono em 1991, e posteriormente do grafeno em 2004, revolucionou a ciência e a tecnologia dos materiais. Novos fenômenos advindos da escala nanométrica foram observados, e com eles propriedades singulares e superlativas, que prometeram um mundo novo de possibilidades. Nanotubos de carbono e grafeno são materiais extremamente leves e flexíveis, que apresentam a maior resistência mecânica e a maior condutividade térmica de todos os materiais conhecidos, com a condutividade elétrica modulável, podendo ir de semicondutores a metálicos (e 
supercondutores em alguns casos), quimicamente inertes, extremamente resistentes à temperatura, possuem alta área superficial, alta transparência (grafeno), e ainda permitem ser funcionalizados com diferentes grupamentos químicos. Todas essas propriedades fazem das chamadas nanoestruturas de carbono materiais fantásticos, únicos, extremamente representativos da nanociência e da nanotecnologia, e com um grande número de aplicações em potencial. Atualmente é muito difícil imaginar um único setor da atividade humana que não tenha possibilidade, de alguma forma, de ser impactado e beneficiado pelos nanotubos de carbono ou grafeno. A princípio toda e qualquer aplicação envolvendo grafite ou negro de fumo poderia ser extremamente melhorada pela substituição por nanotubos ou grafeno, desde que, logicamente, os preços se tornem competitivos, e a tecnologia para substituição bem estabelecida. ${ }^{1,4}$

$\mathrm{O}$ vasto conhecimento científico existente sobre o grafite e as diferentes formas de carbono $\mathrm{sp}^{2}$ serviu de alicerce para que a ciência ao redor dos nanotubos de carbono pudesse ser construída, com a vantagem adicional que essas nanoestruturas surgiram num momento especial da ciência mundial, onde equipamentos de caracterização novos e extremamente sofisticados foram criados, permitindo um aprofundamento indispensável na caracterização desses materiais. E naturalmente, o conhecimento sobre os nanotubos de carbono acelerou sobremaneira o desenvolvimento da ciência e tecnologia ao redor do grafeno.

Inicialmente é preciso deixar claro que "nanotubos de carbono" correspondem a uma família de diferentes materiais, que têm em comum as características já apresentadas anteriormente (folha de grafeno enrolada formando tubos de dimensões nanométricas), mas que diferem entre si com relação ao diâmetro, à forma pela qual essa folha se enrola (conhecido na literatura científica como quiralidade), ao número de camadas concêntricas (paredes simples ou múltiplas), à presença ou não de defeitos na estrutura (e ao tipo específico de defeitos), à presença ou não de grupos funcionais na superfície (e quais são esses grupos), à pureza e ao tipo de impureza presente. Essas características influenciam fortemente nas propriedades dos nanotubos, e devem ser levadas em conta quando se pensa em aplicações. E todas essas características são dependentes do método de preparação dos nanotubos, e consequentemente influenciam no preço final do produto obtido. ${ }^{1,4}$

As mesmas considerações devem ser feitas com relação ao grafeno: não existe um único grafeno. Apesar de se tratar de uma única folha, com espessura atômica, duas folhas empilhadas também possuem propriedades muito interessantes e diferenciadas; três folhas também (por exemplo, no que diz respeito às propriedades eletrônicas, grafeno com três folhas possui bandgap, enquanto uma única folha tem bandgap nulo). Esses materiais são chamados de grafeno, ou grafeno bicamada, tri-camada, tetra-camada, e multi-camada a partir desse ponto. Acima de poucas camadas já não temos mais grafeno, mas sim um grafite (ou nano-grafite); a presença de defeitos na estrutura do grafeno (interrompendo a "colmeia"), a presença de outros grupos funcionais, o tamanho lateral das folhas, o tipo de borda das folhas, correspondem a características que têm influência direta nas propriedades, e novamente, são fortemente dependentes do método de preparação. ${ }^{1,4}$

Um dos grandes equívocos propagados com relação ao grafeno é que basta extraí-lo do grafite, ou seja, basta possuir grafite que se tem acesso ao grafeno. Apesar de ser o bloco construtor do grafite, o grafeno não está simplesmente disponível no grafite. Embora as forças atrativas entre as folhas de grafeno no grafite sejam do tipo van der Waals, estas se tornam extremamente fortes devido ao efeito cooperativo (ou seja, efeito aditivo devido ao número extremamente alto de ligações de van der Waals entre duas folhas de grafeno), e desfazê-las para a obtenção do grafeno não é uma tarefa simples. O trabalho original que abriu as portas para o mundo do grafeno usou de uma esfoliação mecânica para isolar o grafeno do grafite, através de sucessivos peelings no grafite por meio de fitas adesivas. ${ }^{10}$ Esse método produz grafeno de alta qualidade, mas não é escalonável, ou seja, jamais será alternativa para produção de grafeno em larga escala, visando aplicações industriais. Uma outra forma de isolar o grafeno do grafite se dá através de processos de oxidação do grafite (que aumenta a separação entre as folhas através da adição de grupos funcionais oxigenados), seguido da exfoliação do óxido de grafite em água, gerando o óxido de grafeno (GO), seguido da redução química desse material tentando reestabelecer a estrutura do grafeno. ${ }^{11}$ Esse é um método trabalhoso, mas que permite a produção de grande quantidade de material, além de produzir um outro material bidimensional extremamente interessante, o GO. A desvantagem é que o processo total deixa muitos defeitos na estrutura, e a permanência de alguns grupos funcionais oxigenados, produzindo, portanto, um grafeno de baixa qualidade estrutural. Métodos de exfoliação química do grafite (usando moléculas ou íons para separar as folhas no grafite) também são eficientes e escalonáveis, ${ }^{12}$ mas normalmente usam de ultrassom para facilitar o processo, o que acarreta na quebra do tamanho das folhas. Além disso, as amostras são heterogêneas com relação ao número de camadas, e ainda normalmente têm as espécies químicas aderidas na sua superfície. Em resumo: ainda é necessária muita pesquisa básica para extrair o grafeno do grafite em quantidade, qualidade, e produzindo amostras reprodutíveis. Para muitas aplicações, a estratégia bottom up é bastante interessante, que consiste em sintetizar o grafeno a partir de moléculas precursoras. Destacam-se aqui o método CVD (Chemical Vapour Deposition) na qual uma molécula precursora é decomposta em fornos em altas temperaturas, produzindo grafeno sobre substratos metálicos como cobre ou níquel, ${ }^{13}$ ou o método de síntese orgânica, onde através de reações químicas específicas, como por exemplo as reações de acoplamento aromático oxidativo, o grafeno pode ser obtido em condições ambiente. ${ }^{14,15}$

O mercado anual de nanotubos de carbono foi de 4,55 bilhões de dólares em 2018, e é estimado um crescimento para 9,84 bilhões em $2023,{ }^{16}$ com uma demanda crescente em aplicações em compósitos, recobrimentos, sensores, dispositivos de emissão de campo, em armazenamento de hidrogênio, circuito integrado, células solares, baterias de lítio, células a combustível e drug delivery. A capacidade global instalada para a produção e comercialização de nanotubos de carbono do tipo parede simples vem aumentando, com melhora na qualidade, maior controle no tipo de nanotubo e reduzindo os preços. Nanotubos multi-paredes são os mais produzidos, mais utilizados, e mais baratos, mas a capacidade total de produção instalada vem diminuindo nos anos recentes. ${ }^{17}$ Com relação aos nanotubos de paredes múltiplas, embora os custos ainda sejam ligeiramente superiores ao negro de fumo, por exemplo, o ganho em qualidade e a diminuição na quantidade necessária para desempenhar funções específicas os tornam extremamente competitivos. Vários produtos comerciais contendo nanotubos de carbono em sua composição já são amplamente disponíveis no mercado, incluindo compósitos poliméricos para diversos tipos de aplicação (como por exemplo em materiais esportivos de alto desempenho e na indústria automobilística), baterias, eletrodos transparentes, dentre outros.

No que diz respeito ao grafeno, o mercado em 2017 foi estimado em US\$ 42,8 milhões, com uma expectativa de crescimento de $38 \%$ até $2025,{ }^{18}$ com aplicações bastante similares às propostas para os nanotubos de carbono. Várias indústrias têm surgido diretamente envolvidas com grafeno, com interesse em compósitos, dispositivos eletrônicos, sensores, produção e modificação de grafeno e outros materiais bidimensionais, fotônica, membranas, etc. ${ }^{19} \mathrm{O}$ modismo recente em relação ao grafeno, entretanto, deve ser tratado com muita cautela. 
Por exemplo, é preciso atentar para o grande número de amostras comerciais que se tratam, na verdade, de "falso grafeno" (do inglês fake graphene), onde materiais que não têm características do grafeno são erroneamente classificados como tal..$^{20} \mathrm{Um}$ estudo sistemático recente analisou 60 empresas que produzem grafeno (não inclui GO e rGO) no mundo (Américas, Ásia e Europa), e mostrou que grande parte delas vendem um "grafeno falso", de péssima qualidade, consistindo basicamente de grafite. ${ }^{21}$ Indicadores de qualidade como tamanho de folha, integridade estrutural e pureza foram avaliados. Menos de $10 \%$ do material analisado consistia de grafeno com 10 camadas ou menos. Ainda, nenhum dos produtos continha mais de $50 \%$ de grafeno, e todos apresentavam muita contaminação. Nesse estudo, os autores atribuem que essa má qualidade dos produtos comercializados como grafeno pode ser uma das causas pelo lento desenvolvimento das aplicações reais com grafeno. Prevê-se que muitas aplicações emergentes com grafeno estejam fortemente ameaçadas em decorrência dessas amostras falsas. Assim, faz-se necessário a implementação de regulação adequada e protocolos de controle de qualidade que garantam confiabilidade no processo de produção do grafeno.

A importância tecnológica e estratégica dessas duas nanoestruturas de carbono é evidente, e o Brasil tem a possibilidade de acompanhar esse desenvolvimento em nível mundial, se políticas corretas forem adotadas. Temos uma competência instalada de alto nível, dentro das nossas universidades, no que diz respeito aos nanotubos de carbono e grafeno, com uma produção científica extensa e relevante. Ao analisar dados no "Web of Science" em Julho/2019, na área de nanotubos de carbono (pesquisa de tópico com "carbon nanotubes"), no período de 1992-2018, o mundo registrou 142185 publicações, enquanto o Brasil entre 1993-2018, publicou
2275 , o que representa $1,6 \%$ do total no mundo. Isso demonstra um leve aumento em relação a uma análise similar feita em 2013, onde o Brasil na época representava $1,09 \%$ da produção mundial. ${ }^{1}$ A Figura 1A abaixo mostra a distribuição da produção científica no decorrer dos anos, mostrando que ainda vem crescendo no período recente. A porcentagem de crescimento na produção está ilustrada na Figura 1B e observa-se que até 2012, o crescimento era maior no mundo do que no Brasil e nos últimos anos o processo se inverteu. Por exemplo, desde 2016, a produção científica no Brasil cresceu mais do que em relação ao mundo.

Outra análise interessante é se considerar aonde essas pesquisas vêm sendo realizadas. A Figura 2 apresenta as 15 instituições que mais publicaram em nanotubos de carbono no Brasil, no período de 1993-2018. Essas instituições representam 90\% de toda publicação brasileira, e são todas universidades públicas, sendo 13 federais e 2 estaduais de São Paulo. Nota-se ainda que dentre as 15 instituições que mais publicam no Brasil, tem-se cerca de 9\%,67\% e $14 \%$ na região Nordeste, Sudeste e Sul, respectivamente.

No cenário da pesquisa na área de grafeno, que surgiu muito mais recentemente, a participação do Brasil nas publicações também é notória. Segundo o "Web of Science" (pesquisa de tópico com "graphene"), no período entre 2004-2018 tem-se 166806 publicações no mundo todo. O Brasil contribui com $1,25 \%$ na produção mundial, tendo 2103 publicações entre 2001-2018 (entre 2004-2018 tem-se 2095 artigos). Em 2013, a produção do Brasil nessa área em relação ao mundo era de $1,23 \%$. $^{1}$ A Figura $3 \mathrm{~A}$ apresenta a distribuição de publicações por ano no Brasil, comparado com o mundo. Mostra um crescimento mais acentuado comparado com os dados envolvendo nanotubos de carbono, decorrente claramente da sua mais recente
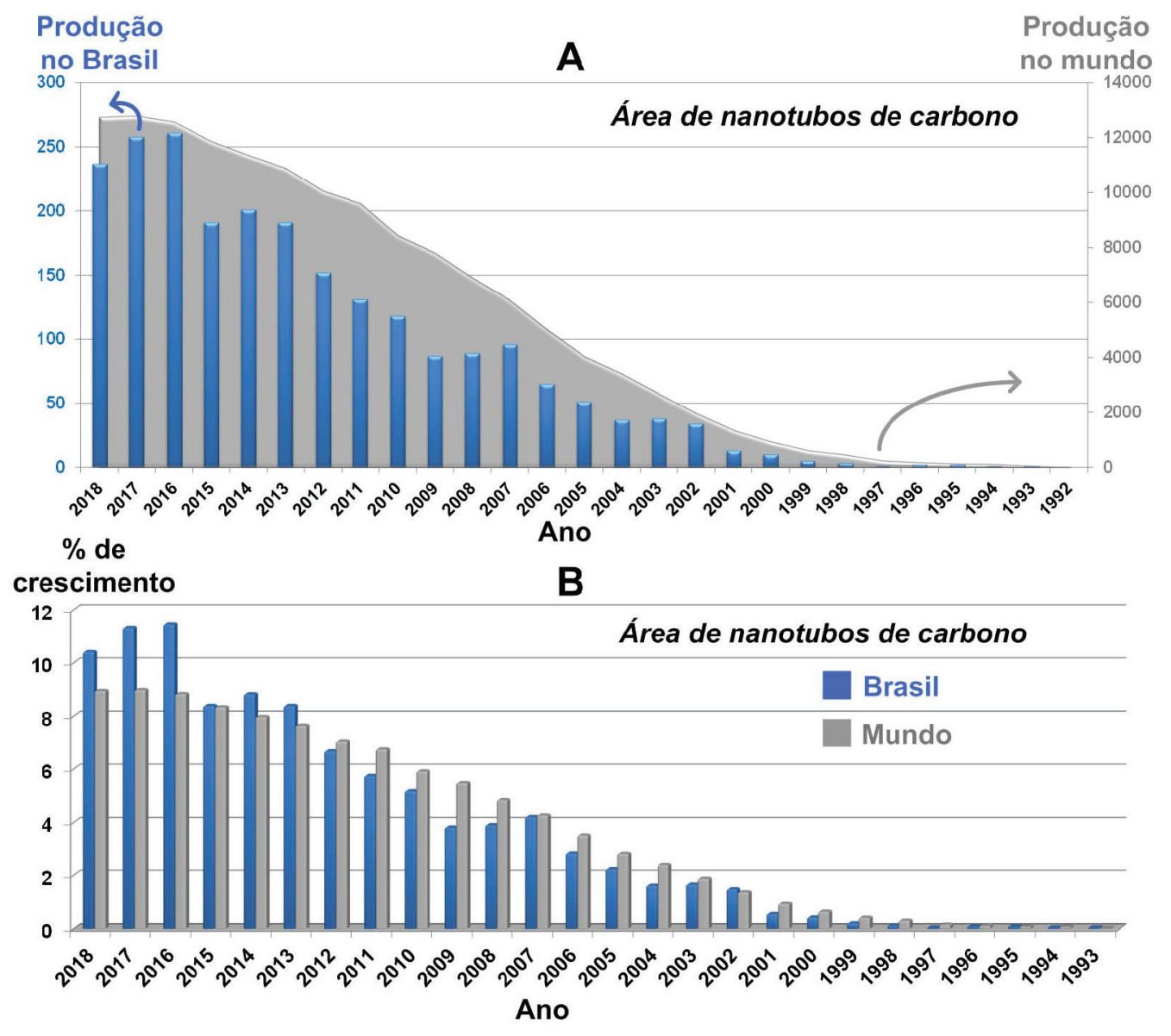

Figura 1. Produção científica na área de nanotubos de carbono, no mundo e no Brasil, obtido pelo "Web of Science" em Julho/2019, dado A) em números totais e B) em porcentagem de crescimento 


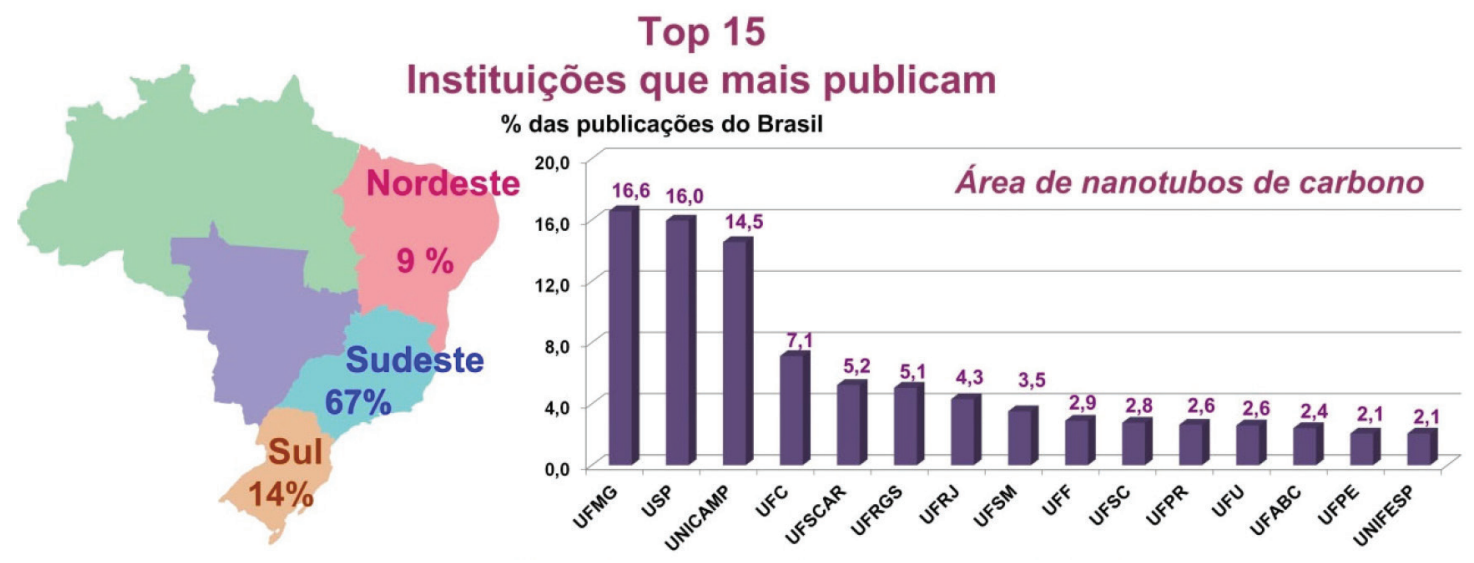

Figura 2. As 15 instituições e sua distribuição por região, que mais publicam na área de nanotubos de carbono no Brasil entre 1993-2018 segundo o "Web of Science" (Julho/2019)

descoberta. A Figura 3B ilustra o crescimento percentual nas publicações na área de grafeno no Brasil e no mundo, evidenciando um perfil maior de crescimento comparado com o perfil observado com nanotubos de carbono. Por exemplo, com nanotubos de carbono, o crescimento passou pouco acima de $10 \%$ ao ano. Já na área de grafeno, tem-se um crescimento que chega a $20 \%$. Ainda, observa-se que o crescimento na produção brasileira com grafeno está mais equiparada com o resto do mundo, quando comparada com a temática de nanotubo de carbono.

Analisando as 15 universidades brasileiras que mais publicam na área de grafeno, sendo 14 universidades públicas e uma privada, apresentado na Figura 4, observa-se que a UNICAMP, USP e UFMG lideram, com cerca de $40 \%$ de toda produção brasileira. Ainda, essas 15 universidades representam $85 \%$ de toda produção brasileira, pouco menos que na área de nanotubos de carbono (Figura 2). Isso significa que a área de grafeno não se concentra só em algumas universidades e tem maior capilaridade. De fato, o percentual que cada universidade contribui é menor na área de grafeno que com nanotubos de carbono. Analisando por região, essas 15 universidades que mais publicam na área estão concentradas na região Nordeste, Sudeste e Sul com $15 \%$, $63 \%$ e $6 \%$, respectivamente. Comparando com a área de nanotubos de carbono, observa-se algumas diferenças. A região sudeste tem

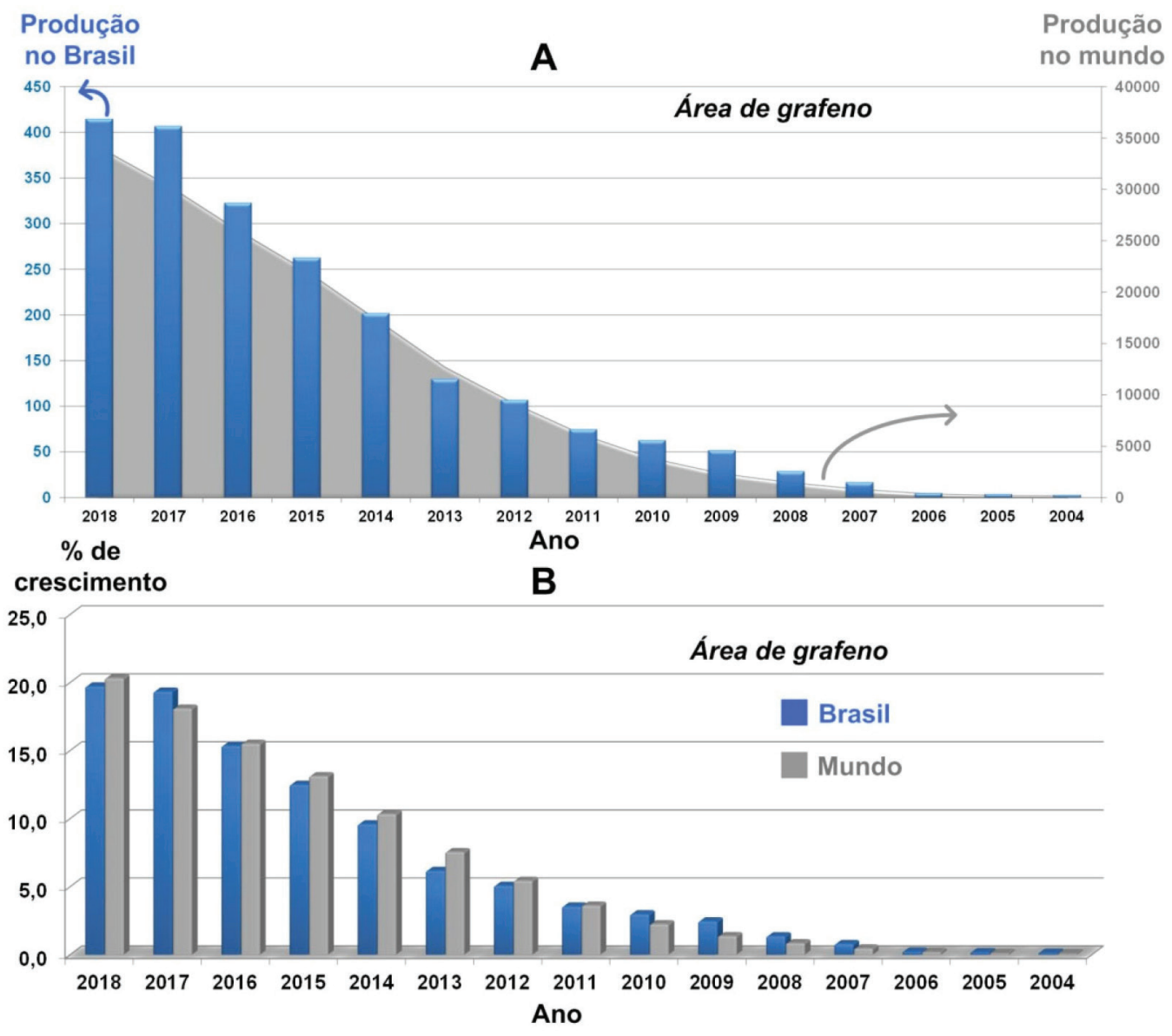

Figura 3. Produção científica na área de grafeno, no mundo e no Brasil, obtido pelo "Web of Science" em Julho/2019, dado A) em números totais e B) porcentagem de crescimento 


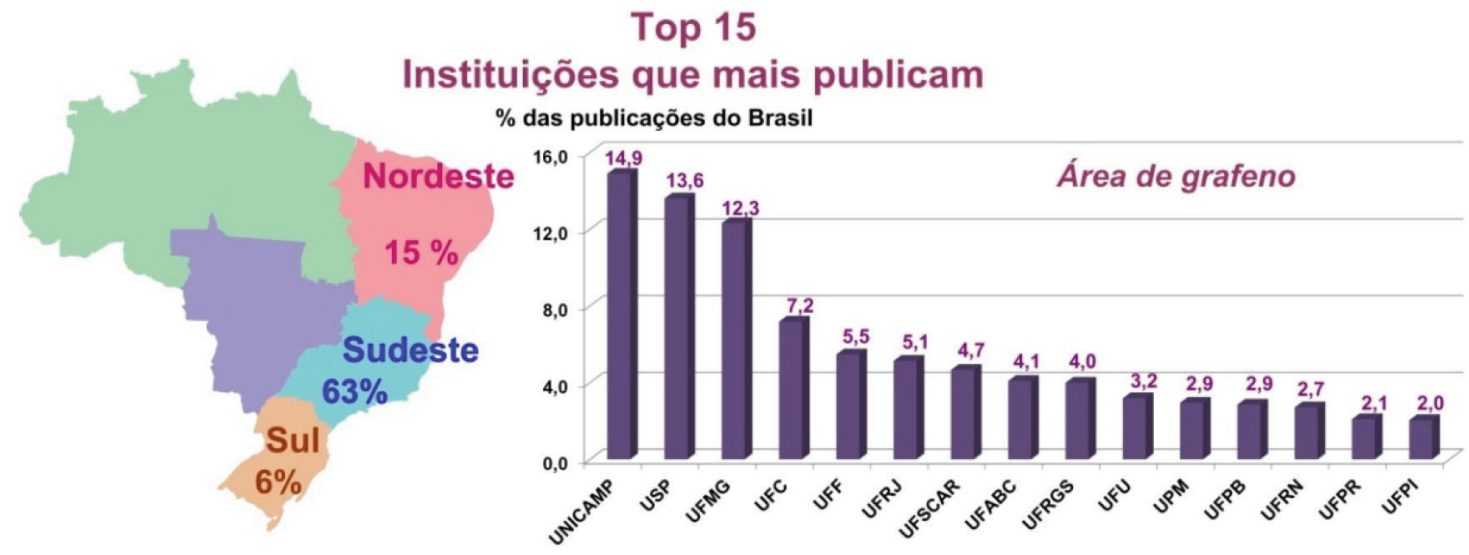

Figura 4. As 15 instituições e sua distribuição por região, que mais publicam na área de grafeno no Brasil entre 1993-2018 segundo o "Web of Science" (Julho/2019)

uma menor participação na área de grafeno, enquanto o Nordeste tem participação maior, distribuído em 4 universidades diferentes ( 2 universidades com nanotubos de carbono). Em contraste, a região sul está representada apenas por 2 universidades, a UFPR e UFRGS (4 universidades no caso de nanotubos de carbono).

A importância da ciência brasileira na área de nanotubos de carbono e de grafeno é fruto também do histórico de organização dos pesquisadores em redes colaborativas, o que certamente auxiliou na consolidação da posição de destaque que o país possui nessas áreas. Entre 2005 e 2010 esteve sob vigência a Rede Nacional de Pesquisa em Nanotubos de Carbono, uma das redes de nanotecnologia financiadas pelo MCT/CNPq, envolvendo 40 pesquisadores de 13 universidades em 8 estados da federação, além da participação de 5 empresas. Entre 2009 e 2016 foi formado o Instituto Nacional de Ciência e Tecnologia em Nanomateriais de Carbono (INCT-Nanocarbono), que foi renovado para o período de 2017-2020, com a participação de 85 pesquisadores, 25 instituições de pesquisa em 9 empresas. ${ }^{22}$ Os encontros anuais do INCT Nanocarbono têm a participação de mais de 270 pesquisadores, incluindo estudantes de pós-graduação e posdocs, o que demonstra a força dessa comunidade no país.

Outro panorama a se analisar na área de nanotubos de carbono e grafeno é na inovação. Analisando dados do Instituto Nacional da Propriedade Industrial (INPI) em Julho/2019, tem-se registrado 173 e 108 patentes depositadas envolvendo nanotubos de carbono e grafeno, respectivamente (busca pela palavra chave no resumo em Julho/2019). Esses números são bem maiores que os reportados em 2013, que mostrou apenas 57 e 3 patentes com nanotubos de carbono e grafeno, respectivamente. ${ }^{1}$ Isso evidencia como esses materiais possuem grande potencial de inovação.

Finalmente, merecem destaque duas grandes iniciativas no Brasil, especificamente no estado de Minas Gerais, já avançando na direção do setor produtivo. Em 2019 foi inaugurada a sede definitiva do Centro de Tecnologia em Nanomateriais e Grafeno (CTNano), que tem como objetivos ser uma ponte entre a academia e a indústria. Hoje o CTNano produz nanotubos de carbono e grafeno em escala, além de projetos avançados envolvendo cimento nanoestruturado e nanocompósitos poliméricos. O centro é especializado ainda na caracterização e metrologia para controle de qualidade, no desenvolvimento de protocolos pra o uso seguro da nanotecnologia para o meio ambiente e a saúde. ${ }^{23}$ O CTNano nasceu de uma demanda específica da Petrobras em 2014, e foi consolidado com apoio das empresas Petrobras e InterCement, do Banco Nacional de Desenvolvimento Econômico e Social (BNDES), da Financiadora de Estudos e Projetos (FINEP), da Agência Nacional do Petróleo, Gás Natural e Biocombustíveis (ANP), da Fundação de Amparo à Pesquisa do Estado de Minas Gerais (FAPEMIG), do
BH-TEC, da UFMG e da Fundação de Desenvolvimento da Pesquisa (FUNDEP). ${ }^{23}$

E também em Minas Gerais, o projeto MGgrafeno é uma parceria entre a Companhia de Desenvolvimento de Minas Gerais CODEMGE, o Centro de Desenvolvimento da Tecnologia Nuclear - CDTN e a UFMG, criada com o objetivo de desenvolver um processo escalável e de baixo custo para produção de grafenos a partir da esfoliação química do grafite natural cristalino, além da demonstração de algumas aplicações atraentes que utilizem os nanomateriais produzidos. O projeto objetiva agregar valor ao grafite e criar uma cadeia de negócios inovadores em torno de suas aplicações após processamento. Atualmente, um processo piloto eficiente e sustentável de produção de grafenos de poucas camadas já opera em instalações do CDTN, gerando basicamente dois nanomateriais: o grafeno e as nanoplacas de grafeno, com capacidade instalada de aproximadamente $150 \mathrm{~kg} /$ ano e 1,2 ton/ano, respectivamente. Protocolos avançados de caracterização físico-química de grafenos, avaliação de riscos e tratamento de resíduos da produção desses nanomateriais de carbono são também produtos do projeto. ${ }^{24}$

\section{CONSIDERAÇÕES FINAIS E CONCLUSÕES}

Os diferentes alótropos do carbono são materiais extremamente importantes do ponto de vista tecnológico, industrial, econômico e social. Essa alta relevância não se restringe aos quatro materiais abordados nesse texto, mas se estende à grande família de substâncias elementares baseadas no carbono, como o diamante para aplicações industriais, o carvão para geração de energia, e o fulerenos em aplicações em medicina ou células solares. Pode-se afirmar sem sombra de dúvidas que o carbono é um elemento químico extremamente estratégico para o desenvolvimento e soberania do país.

$\mathrm{O}$ advento da nanociência e nanotecnologia trouxe a reboque promessas de uma verdadeira revolução nos costumes e na forma pela qual nos relacionamos com as coisas, e novamente o carbono, com suas duas nanoestruturas fantásticas - os nanotubos e o grafeno - teve um papel central nessa expectativa. Passado o momento de euforia, essas nanoestruturas vêm se consolidando como materiais estratégicos reais, com um mercado em crescimento, e com novas demandas ainda a serem conquistadas. O Brasil possui capacidade instalada nas Universidades e Institutos de Pesquisa para atender e fomentar demandas na área, e pode ser competitivo em nível mundial. Essa capacidade não pode correr o risco de ser destruída, por ausência e/ou cortes nos investimentos. Aliás, a receita é antiga, conhecida, e extremamente eficiente: não há crescimento, desenvolvimento e soberania de uma nação sem um investimento público robusto e 
continuado em ciência, tecnologia e educação. O Brasil conhece os caminhos, basta optar pelo correto.

\section{AGRADECIMENTOS}

Os autores agradecem aos editores desse NE pelo convite; ao CNPq, CAPES, UFPR, L'Oréal-UNESCO-ABC, PhosAgro/ UNESCO/IUPAC e INCT-Nanocarbono pelo apoio financeiro.

\section{REFERENCIAS}

1. Zarbin, A. J. G.; Oliveira, M. M.; Quim. Nova 2013, 36, 1533.

2. Matos, C. F.; Zarbin, A. J. G.; Galembeck, F. Em Carbon-based nanofillers and their rubber nanocomposites: fundamentals and applications; Yaragalla, S., Mishra, R. K., Thomas, S., Kalarikkal, N., Maria, H. J., eds.; Elsevier Ltd.: Amsterdã, 2019, cap. 1.

3. Geim, A. K.; Novoselov, K. S.; Nat Mater. 2007, 6, 183.

4. Tanaka, K.; Iijima, S.; Carbon nanotubes and graphene, Elsevier Ltd.: Amsterdã, 2014.

5. Lima, T. M.; Neves, C. A. R.; Sumário Mineral, Departamento Nacional de Produção Mineral, Brasília, 2018.

6. U. S. Geological Survey, 2019, Mineral commodity summaries 2019: U. S. Geological Survey, 200 p., https://doi.org/10.3133/70202434.

7. https://www.ceresana.com/en/market-studies/chemicals/carbon-black, acessada em novembro 2019.

8. Donnet, J. B.; Carbon black: science and technology, CRC Press: Boca Raton, 1993.

9. Revista Borracha Atual, Edição 139, 2018.

10. Novoselov, K. S.; Geim, A. K.; Morozov, S. V.; Jiang, D.; Zhang, Y.; Dubonos, S. V.; Grigorieva, I. V.; Firsov, A. A.; Science 2004, 306, 666.
11. Mehl, H.; Matos, C. F.; Neiva, E. G.; Domingues, S. H.; Zarbin, A. J. G.; Quim. Nova 2014, 37, 1639.

12. Feng, H.; Wu, Y.; Li, J.; Small 2014, 10, 2233.

13. Li, Z.; Wu, P.; Wang, C.; Fan, X.; Zhang, W.; Zhai, X.; Zeng, C.; Li, Z.; Yang, J.; Hou, J.; ACS Nano 2011, 5, 3385.

14. Salvatierra, R. V.; Souza, V. H. R.; Matos, C. F.; Oliveira, M. M.; Zarbin, A. J. G.; Carbon 2015, 93, 924.

15. Lopes, L. C.; Silva, L. C.; Vaz, B. G.; Oliveira, A. R. M.; Oliveira, M. M., Rocco, M. L. M.; Orth, E. S.; Zarbin, A. J. G.; Chem. Sci. 2018, 9 , 7297.

16. https://www.marketsandmarkets.com/Market-Reports/carbonnanotubes-139.html, acessada em novembro 2019.

17. https://www.globenewswire.com/news-release/2019/06/11/1866732/0/ en/Global-Carbon-Nanotubes-Markets-2018-2019-2019-2030.html, acessada em novembro 2019.

18. https://www.grandviewresearch.com/industry-analysis/grapheneindustry, acessada em novembro 2019.

19. Correia, A.; Graphene companies catalogue \& research centers, Phanton Fundation: Madrid, 2019.

20. Bøggild, P.; Nature 2018, 562, 502.

21. Kauling, A. P.; Seefeldt, A. T.; Pisoni, D. P.; Pradeep, R. C.; Bentini, R.; Oliveira, R. V. B.; Novoselov, K. S.; Castro Neto, A. H.; Adv. Mater. 2018, 30, 1803784.

22. http://inct.cnpq.br/web/inct-nanocarbono, acessada em novembro 2019.

23. http://www.sbfisica.org.br/v1/home/index.php/pt/acontece/885-centrode-tecnologia-em-nanomateriais-e-grafeno-inaugura-nova-sede, acessada em novembro 2019.

24. http://www.cdtn.br/ultimas-noticias/121-newsletter/362-planta-pilotode-grafeno-em-minas-impulsiona-desenvolvimento-industrial-emnanotecnologia, acessada em novembro 2019. 